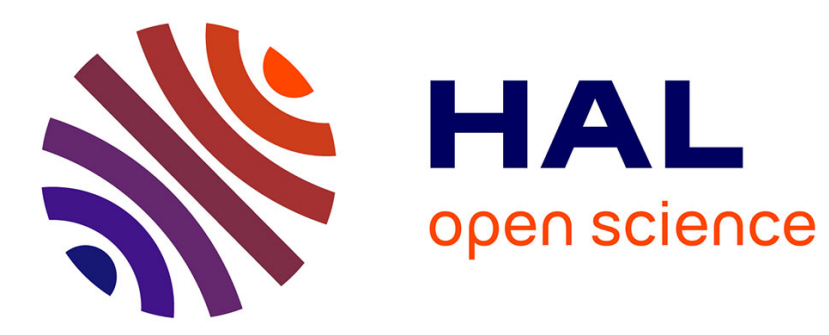

\title{
A multi-resolution approach to common fate-based audio separation
}

\author{
Fatemeh Pishdadian, Bryan Pardo, Antoine Liutkus
}

\section{To cite this version:}

Fatemeh Pishdadian, Bryan Pardo, Antoine Liutkus. A multi-resolution approach to common fatebased audio separation. 42nd International Conference on Acoustics, Speech and Signal Processing (ICASSP), Mar 2017, New Orleans, United States. hal-01515951

\section{HAL Id: hal-01515951 \\ https://hal.inria.fr/hal-01515951}

Submitted on 28 Apr 2017

HAL is a multi-disciplinary open access archive for the deposit and dissemination of scientific research documents, whether they are published or not. The documents may come from teaching and research institutions in France or abroad, or from public or private research centers.
L'archive ouverte pluridisciplinaire HAL, est destinée au dépôt et à la diffusion de documents scientifiques de niveau recherche, publiés ou non, émanant des établissements d'enseignement et de recherche français ou étrangers, des laboratoires publics ou privés. 


\section{A MULTI-RESOLUTION APPROACH TO COMMON FATE-BASED AUDIO SEPARATION}

\author{
Fatemeh Pishdadian, Bryan Pardo * \\ Northwestern University, USA \\ \{fpishdadian@u.,pardo@\}northwestern.edu
}

\author{
Antoine Liutkus \\ Inria, speech processing team, France \\ antoine.liutkus@inria.fr
}

\begin{abstract}
We propose the Multi-resolution Common Fate Transform (MCFT), a signal representation that increases the separability of audio sources with significant energy overlap in the time-frequency domain. The MCFT combines the desirable features of two existing representations: the invertibility of the recently proposed Common Fate Transform (CFT) and the multi-resolution property of the cortical stage output of an auditory model. We compare the utility of the MCFT to the CFT by measuring the quality of source separation performed via ideal binary masking using each representation. Experiments on harmonic sounds with overlapping fundamental frequencies and different spectro-temporal modulation patterns show that ideal masks based on the MCFT yield better separation than those based on the CFT.
\end{abstract}

Index Terms - Audio source separation, Multi-resolution Common Fate Transform,

\section{INTRODUCTION}

Audio source separation is the process of estimating $n$ source signals given $m$ mixtures. It facilitates many applications, such as automatic speaker recognition in a multi-speaker scenario [1, 2], musical instrument recognition in polyphonic audio [3], music remixing [4], music transcription [5], and upmixing of stereo recordings to surround sound $[6,7]$.

Many source separation algorithms share a weakness in handling the time-frequency overlap between sources. This weakness is caused or exacerbated by their use of a timefrequency representation, typically the short-time Fourier transform (STFT), for the audio mixture. For example, the Degenerate Un-mixing and Estimation Technique (DUET) $[8,9]$ clusters time-frequency bins based on attenuation and delay relationships between STFTs of the two channels. If multiple sources have energy in the same time-frequency bin, the performance of DUET degrades dramatically, due to the inaccurate attenuation and delay estimates. Kernel Additive Modeling (KAM) [10, 11] uses local proximity of points belonging to a single-source. While the formulation of KAM does not make any restricting assumptions about the

*Thanks to support from National Science Foundation Grant 1420971. audio representation, the published work uses proximity measures defined in the time-frequency domain. This can result in distortion if multiple sources share a time-frequency bin. Non-negative Matrix Factorization (NMF) [12] and Probabilistic Latent Component Analysis (PLCA) [13] are popular spectral decomposition-based source separation methods applied to the magnitude spectrogram. The performance of both degrades as overlap in the time-frequency domain increases.

Overlapping energy may be attenuated in better representations. According to the common fate principle [14], spectral components moving together are more likely to be grouped into a single sound stream. A representation that makes common fate explicit (e.g. as one of the dimensions) would facilitate separation, since the sources would better form separate clusters, even when overlapping in time and frequency.

There has been some recent work in the development of richer representations to facilitate separation of sounds with significant time-frequency energy overlap. Stöter et al. [15] proposed a new audio representation, named the Common Fate Transform (CFT). This 4-dimensional representation is computed from the complex STFT of an audio signal by first dividing it into a grid of overlapping patches (2D windowing) and then analyzing each patch by the 2D Fourier transform. The CFT was shown to be promising for the separation of sources with the same pitch (unison) and different modulation. However, they use a fixed-size patch for the whole STFT. This limits the spatial frequency resolution, affecting the separation of streams with close modulation patterns.

The auditory model proposed by Chi et al. [16] emulates important aspects of the cochlear and cortical processing stages in the auditory system. It uses a bank of 2-dimensional, multi-resolution filters to capture and represent spectro-temporal modulation. This approach avoids the fixed-size windowing issue. Unfortunately, creation of the representation involves non-linear operations and removing phase information. This makes perfect invertibility to the time domain impossible. Thus, using this representation for source separation (e.g. Krishnan et al. [17]) requires building masks in the time-frequency domain, where it is possible to reconstruct the time-domain signal. However, masking in time-frequency eliminates much of the benefit of explicitly representing spectro-temporal modulation, since time-frequency overlap between sources remains a problem. 
Here, we propose the Multi-resolution Common Fate Transform (MCFT), which combines the invertibility of the CFT with the multi-resolution property of Chi's auditorymodel output. We compare the efficacy of the CFT and the MCFT for source separation on mixtures with considerable time-frequency-domain overlap (e.g. unison mixtures of music instruments with different modulation patterns).

\section{PROPOSED REPRESENTATION}

We now give brief overviews of the Common Fate Transform [15] and Chi's auditory model [16]. We then propose the Multi-resolution Common Fate Transform (MCFT), which combines the invertibility of the CFT with the multiresolution property of Chi's auditory-model output.

\subsection{Common Fate Transform}

Let $x(t)$ denote a single channel time-domain audio signal and $X(\omega, \tau)=|X(\omega, \tau)| e^{j \angle X(\omega, \tau)}$ its complex timefrequency-domain representation. Here, $\omega$ is frequency, $\tau$ time-frame, $|$.$| is the magnitude operator, and \angle($.$) is the$ phase operator. In the original version of CFT [15], $X(\omega, \tau)$ is assumed to be the STFT of a signal, computed by windowing the time-domain signal and taking the discrete Fourier transform of each frame.

In the following step, a tensor is formed by $2 \mathrm{D}$ windowing of $X(\omega, \tau)$ with overlapping patches of size $L_{\omega} \times L_{\tau}$ and computing the 2D Fourier transform of each patch. Patches are overlapped along both frequency and time axes. To keep the terminology consistent with the auditory model (see Section 2.2), the 2D Fourier transform domain will be referred to as the scale-rate domain throughout this paper. We denote the 4-dimensional output representation of CFT by $Y(s, r, \Omega, T)$, where $(s, r)$ denotes the scale-rate coordinate pair and $(\Omega, T)$ gives the patch centers along the frequency and time axes. As mentioned earlier, the choice of patch dimensions has a direct impact on the separation results. Unfortunately, no general guideline for choosing the patch size was proposed in [15].

All processes involved in the computation of CFT are perfectly invertible. The single-sided complex STFT, $X(\omega, \tau)$, can be reconstructed from $Y(s, r, \Omega, T)$ by taking the $2 \mathrm{D}$ inverse Fourier transform of all patches and then performing 2D overlap and add of the results. The time-signal, $x(t)$, can then be reconstructed by performing 1D inverse Fourier transform of each frame followed by 1D overlap and add.

\subsection{The Auditory Model}

The computational model of early and central stages of the auditory system proposed in Chi et al. [16] (see also [18]) yields a multi-resolution representation of spectro-temporal features that are important in sound perception. The first stage of the model, emulating the cochlear filter-bank, performs spectral analysis on the input time-domain audio signal. The analysis filter-bank includes 128 overlapping constant-Q bandpass filters. The center frequencies of the filters are logarithmically distributed, covering around 5.3 octaves. To replicate the effect of processes that take place between the inner ear and midbrain, more operations including high-pass filtering, nonlinear compression, half-wave rectification, and integration are performed on the output of the filter bank. The output of the cochlear stage, termed auditory spectrogram, is approximately $|X(\omega, \tau)|$, with a logarithmic frequency scale.

The cortical stage of the model emulates the way the primary auditory cortex extracts spectro-temporal modulation patterns from the auditory spectrogram. Modulation parameters are estimated via a bank of 2D bandpass filters, each tuned to a particular modulation pattern. The 2-dimensional (time-frequency-domain) impulse response of each filter is termed the Spectro-Temporal Receptive Field (STRF). An STRF is characterized by its spectral scale (broad or narrow), its temporal rate (slow or fast), and its moving direction in the time-frequency plane (upward or downward). Scale and rate, measured respectively in cycles per octave and $\mathrm{Hz}$, are the two additional dimensions (besides time and frequency) in this 4-dimensional representation.

We denote an STRF that is tuned to the scale-rate parameter pair $(S, R)$ by $h(\omega, \tau ; S, R)$. Its 2D Fourier transform is denoted by $H(s, r ; S, R)$, where $(s, r)$ indicates the scalerate coordinate pair and $(S, R)$ determines the center of the 2D filter. STRFs are not separable functions of frequency and time ${ }^{1}$. However, they can be modeled as quadrant separable, meaning that their 2D Fourier transforms are separable functions of scale and rate in each quadrant of the transform space. The first step in obtaining the filter impulse response (STRF) is to define the spectral and temporal seed functions. The spectral seed function is modeled as a Gabor-like filter

$$
f(\omega ; S)=S\left(1-2(\pi S \omega)^{2}\right) e^{-(\pi S \omega)^{2}},
$$

and temporal seed function as a gammatone filter.

$$
g(\tau ; R)=R(R \tau)^{2} e^{-\beta R \tau} \sin (2 \pi R \tau)
$$

Equations (1) and (2) demonstrate that filter centers in the scale-rate domain, $S$ and $R$, are in fact the dilation factors of the Gabor-like and gammatone filters in the time-frequency domain. The time constant of the exponential term, $\beta$, determines the dropping rate of the temporal envelop. Note that the product of $f$ and $g$ can only model the spectral width and temporal velocity of the filter, but it does not present any upor down-ward moving direction (due to the inseparability of STRFs in the time-frequency domain). Thus, in the next step, the value of $H$ over all quadrants is obtained as the product of the 1D Fourier transform $\mathcal{F} \mathcal{T}_{1 D}$ of the seed functions, i.e.

$$
H(s, r ; S, R)=F(s ; S) \cdot G(r ; R),
$$

${ }^{1} h(\omega, \tau)$ is called a separable function of $\omega$ and $\tau$ if it can be stated as $h(\omega, \tau)=f(\omega) \cdot g(\tau)$. 
where

$$
\begin{aligned}
& F(s ; S)=\mathcal{F} \mathcal{T}_{1 D}\{f(\omega ; S)\}, \\
& G(r ; R)=\mathcal{F} \mathcal{T}_{1 D}\{g(\tau ; R)\} .
\end{aligned}
$$

The scale-rate-domain response of the upward moving filter, denoted by $H^{\Uparrow}(s, r ; S, R)$, is obtained by zeroing out the first and fourth quadrants: $(s>0, r>0)$ and $(s<0, r<0)$. The response of the downward filter, $H^{\Downarrow}(s, r ; S, R)$, is obtained by zeroing out the second and third quadrants: $(s>0, r<$ $0)$ and $(s<0, r>0)$. Finally, the impulse responses are computed as

$$
\begin{aligned}
& h^{\Uparrow}(\omega, \tau ; S, R)=\Re\left\{\mathcal{I F}_{2} \mathcal{T}_{2 D}\left\{H^{\Uparrow}(s, r ; S, R)\right\}\right\}, \\
& h^{\Downarrow}(\omega, \tau ; S, R)=\Re\left\{\mathcal{I F}_{2} \mathcal{T}_{2 D}\left\{H^{\Downarrow}(s, r ; S, R)\right\}\right\},
\end{aligned}
$$

where $\Re\{$.$\} is the real part of a complex value, and \mathcal{I} \mathcal{F} \mathcal{T}_{2 D}\{$. is the $2 \mathrm{D}$ inverse Fourier transform. The 4-dimensional output of the cortical stage is generated by convolving the auditory spectrogram with a bank of STRFs. Note, however, that filtering can be implemented more efficiently in the scale-rate domain. We denote this representation by $Z(S, R, \omega, \tau)$, where $(S, R)$ gives the filter centers along the scale and rate axes. Figure 1 shows an upward moving STRF with a scale of 1 cycle per octave, and a rate of $4 \mathrm{~Hz}$.

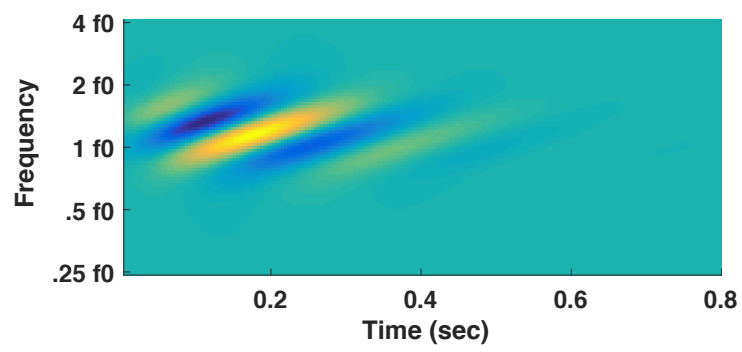

Fig. 1. An upward moving STRF, $h \Uparrow(\omega, \tau ; S=1, R=4)$. The frequency is displayed on a logarithmic scale based on a reference frequency $f_{0}$.

\subsection{Multi-resolution Common Fate Transform}

We address the invertibility issue, caused by the cochlear analysis block of the auditory model, through replacing the auditory spectrogram by a complex time-frequency representation with log-frequency resolution. We use an efficient and perfectly reconstructable implementation of CQT, proposed by Schörkhuber et al. [19]. The new 4-dimensional representation, denoted by $\hat{Z}(S, R, \omega, \tau)$, is computed by applying the cortical filter-bank of the auditory model to the complex CQT of the audi to signal. Note that the time-frequency representation can be reconstructed from $\hat{Z}(S, R, \omega, \tau)$ by inverse filtering as

$$
\tilde{X}(\omega, \tau)=\mathcal{I F}_{2 D}\left\{\frac{\sum_{S, R}^{\Uparrow \Downarrow} \hat{z}(s, r ; S, R) H^{*}(s, r ; S, R)}{\sum_{S, R}^{\Uparrow \Downarrow}|H(s, r ; S, R)|^{2}}\right\},
$$

where $*$ denotes complex conjugate, $\hat{z}(s, r ; S, R)$ is the 2D Fourier transform of $\hat{Z}(\omega, \tau ; S, R)$ for a particular $(S, R)$, and $\sum_{S, R}^{\Uparrow \Downarrow}$ means summation over the whole range of $(S, R)$ values and all up-/down-ward filters. The next modification we make to improve the source separation performance is modulating the filter bank with the phase of the input mixture. We know that components of $|X(\omega, \tau)|$ in the scale-rate domain are shifted according to $\angle X(\omega, \tau)$. Assuming linear phase relationship between harmonic components of a sound, and hence linear shift in the transform domain, we expect to achieve better separation by using modulated filters, i.e. filters with impulse responses equal to $h(\omega, \tau ; S, R) e^{j \angle X(\omega, \tau)}$.

\section{EXPERIMENTS}

In this section we compare the separability provided by the CFT and MCFT for mixtures of instrumental sounds playing in unison, but with different modulation patterns. For a quick comparison, an overview of the computation steps in the CFT and MCFT approaches is presented in Table 1.

\subsection{Dataset}

The main point of our experiments is to demonstrate the efficacy of the overall 4-dimensional representation in capturing amplitude/frequency modulation. We do not focus on the difference in the frequency resolution of STFT and CQT over different pitches or octaves. Thus, we restrict our dataset to a single pitch, but include a variety of instrumental sounds. This approach is modeled on the experiments in the publication where our baseline representation (the CFT) was introduced [15]. There, all experiments were conducted on unison mixtures of note $\mathrm{C} 4$. In our work, all samples except one are selected from the Philharmonia Orchestra dataset ${ }^{2}$.

This dataset had the most samples of note D4 $(293.66 \mathrm{~Hz})$, which is close enough to $\mathrm{C} 4$ to let us use the same transform parameters as in [15]. Samples were played by 7 different instruments (9 samples in total): contrabassoon (minor trill), bassoon (major trill), clarinet (major and minor trill), saxophone (major and minor trill), trombone (tremolo), violin (vibrato), and a piano sample recorded on a Steinway grand. All samples are 2 seconds long and are sampled at $22050 \mathrm{~Hz}$. Mixtures of two sources were generated from all combinations of the 9 recordings ( 36 mixtures in total).

\subsection{CFT and MCFT}

To be consistent with experiments used for the baseline CFT [15], the STFT window length and overlap were set to $\simeq 23$ ms (512 samples) and 50\%. The default patch size (based on [15]) was set to $L_{\omega} \simeq 172.3 \mathrm{~Hz}$ (4 bins ), and $L_{\tau} \simeq 0.74$ sec (64 frames). There was 50\% overlap between patches in both dimensions. We also studied the effect of patch size on

\footnotetext{
${ }^{2}$ www.philharmonia.co.uk
} 


\begin{tabular}{c|c|c|c|c}
\hline Method & Input & Computation Steps & Output \\
\hline CFT & $x(t)$ & STFT $\rightarrow$ 2D-windows centered at $(\Omega, T) \rightarrow \mathcal{F T}_{2 D}$ & $Y(s, r, \Omega, T)$ \\
MCFT & $x(t)$ & $\mathrm{CQT} \rightarrow \mathcal{F T}_{2 D} \rightarrow$ 2D-filters centered at $(S, R) \rightarrow \mathcal{I F}_{2 D}$ & $\hat{Z}(S, R, \omega, \tau)$ \\
\hline
\end{tabular}

Table 1. An overview of the computation steps in CFT and MCFT.

separation, using a grid of values including all combinations of $L_{\omega} \in\{2,4,8\}$ and $L_{\tau} \in\{32,64,128\}$. We present the results for the default, the best, and the worst patch sizes.

We use the MATLAB toolbox in [19] to compute CQTs in our representation. The CQT minimum frequency, maximum frequency, and frequency resolution are respectively $\simeq 65.4 \mathrm{~Hz}$ (note $\mathrm{C} 2)$ and $\simeq 2.09 \mathrm{kHz}$ (note $\mathrm{C} 7$ ), and 24 bins per octave. The spectral filter bank, $F(s ; S)$, include a low pass filter at $S=2^{-3}$ (cyc/oct), 6 band-pass filters at $S=2^{-2}, 2^{-1}, \ldots, 2^{3}$ (cyc/oct), and a high-pass filter at $S=2^{3.5}$ (cyc/oct). The temporal filter bank, $G(r ; R)$, include a low-pass filters at $R=2^{-3} \mathrm{~Hz}, 16$ band-pass filters at $R=2^{-2.5}, 2^{-2}, 2^{-1.5}, \ldots, 2^{5} \mathrm{~Hz}$, and a high-pass filter at $R=2^{6.25} \mathrm{~Hz}$. Each 2D filter response, $H(s, r ; S, R)$, obtained as the product of $F$ and $G$ is split into two analytic filters (see Section 2.2). The time constant of the temporal filter, $\beta$, is set to 1 for the best performance. We have also provided a MATLAB implementation of the method ${ }^{3}$.

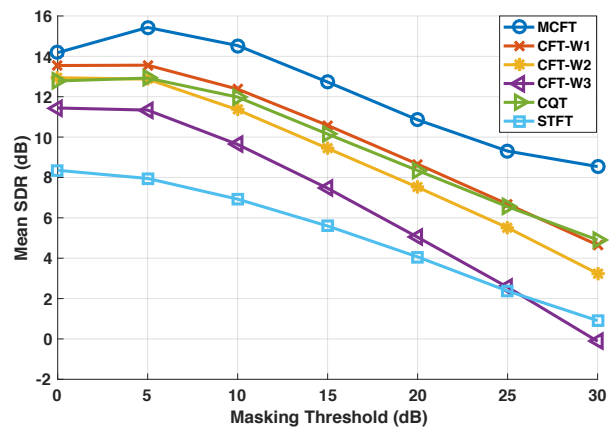

Fig. 2. Mean SDR for $2 \mathrm{D}$ and $4 \mathrm{D}$ representations versus masking threshold. 3 out of 9 patch sizes used in CFT computation are shown: $W_{1}(2 \times 128)$ (best), $W_{2}(4 \times 64)$ (default), and $W_{3}(8 \times 32)$ (worst).

\subsection{Evaluation via Ideal Binary Masking}

To evaluate representations based on the amount of separability they provide for audio mixtures, we construct an ideal binary mask for each source in the mixture. The ideal binary mask assigns a 1 to any point in the representation of the mixture where the ratio of the energy from the target source to the energy from all other sources exceeds a masking threshold. Applying the mask and then returning the signal to the time domain creates a separation whose quality depends only

\footnotetext{
${ }^{3}$ https://github.com/interactiveaudiolab/MCFT
}

on the separability of the mixture when using the representation in question.

We compute the ideal binary mask for each source, in each representation, for a range of threshold values (e.g. $0 \mathrm{~dB}$ to $30 \mathrm{~dB}$ ). We compare separation using our proposed representation (MCFT) to three variants of the baseline representation (CFT), each with a different 2D window size applied to the STFT. We also perform masking and separation using two time-frequency representations: CQT and STFT.

Separation performance is evaluated via the BSS-Eval [20] objective measures: SDR (lower bound on separation performance), SIR, and SAR. Mean SDR over the whole dataset is used as a measure of separability for each threshold value. Figure 2 shows mean SDR values at different masking thresholds. MCFT strictly dominates all other representations at all thresholds. MFCT also shows the slowest dropping rate as a function of threshold. The values of objective measures, averaged over all samples and all thresholds are presented in Table 2, for STFT, CQT, CFT- $W_{1}$ (best patch size), and MCFT. CFT- $W_{1}$ shows an improvement of $4.8 \mathrm{~dB}$ in mean SDR over STFT, but its overall performance is very close to CQT. MCFT improves the mean SDR by $2.5 \mathrm{~dB}$ over CQT and by $2.2 \mathrm{~dB}$ over CFT- $W_{1}$.

\begin{tabular}{|c||c|c|c|}
\hline Method & SDR & SIR & SAR \\
\hline \hline STFT & $5.2 \pm 4.9$ & $20.8 \pm 5.1$ & $5.7 \pm 5.2$ \\
\hline CQT & $9.7 \pm 5.4$ & $23.4 \pm 5.4$ & $10.2 \pm 5.7$ \\
\hline CFT- $W_{1}$ & $10.0 \pm 4.9$ & $24.4 \pm 4.7$ & $10.4 \pm 5.2$ \\
\hline MCFT & $12.2 \pm 3.9$ & $24.1 \pm 5.1$ & $13.2 \pm 4.7$ \\
\hline
\end{tabular}

Table 2. BSS-Eval measures, mean \pm standard deviation over all samples and all thresholds.

\section{CONCLUSION}

We presented MCFT, a representation that explicitly represents spectro-temporal modulation patterns of audio signals, facilitating separation of signals that overlap in timefrequency. This representation is invertible back to time domain and has multi-scale, multi-rate resolution. Separation results on a dataset of unison mixtures of musical instrument sounds show that it outperforms both common timefrequency representations (CQT, STFT) and a recently proposed representation of spectro-temporal modulation (CFT). MCFT is a promising representation to use in combination with state-of-the-art source separation methods that currently use time-frequency representations. 


\section{REFERENCES}

[1] M. Cooke, J. R. Hershey, and S. J. Rennie, "Monaural speech separation and recognition challenge," Computer Speech \& Language, vol. 24, no. 1, pp. 1-15, 2010.

[2] S. Haykin and Z. Chen, "The cocktail party problem," Neural computation, vol. 17, no. 9, pp. 1875-1902, 2005.

[3] T. Heittola, A. Klapuri, and T. Virtanen, "Musical instrument recognition in polyphonic audio using source-filter model for sound separation.," in ISMIR, pp. 327-332, 2009.

[4] J. F. Woodruff, B. Pardo, and R. B. Dannenberg, "Remixing stereo music with score-informed source separation.," in ISMIR, pp. 314-319, 2006.

[5] M. D. Plumbley, S. A. Abdallah, J. P. Bello, M. E. Davies, G. Monti, and M. B. Sandler, "Automatic music transcription and audio source separation," Cybernetics \&Systems, vol. 33, no. 6, pp. 603-627, 2002.

[6] S.-W. Jeon, Y.-C. Park, S.-P. Lee, and D.-H. Youn, "Robust representation of spatial sound in stereo-tomultichannel upmix," in Audio Engineering Society Convention 128, Audio Engineering Society, 2010.

[7] D. Fitzgerald, "Upmixing from mono-a source separation approach," in 2011 17th International Conference on Digital Signal Processing (DSP), pp. 1-7, IEEE, 2011.

[8] S. Rickard, "The duet blind source separation algorithm," Blind Speech Separation, pp. 217-237, 2007.

[9] S. Rickard and O. Yilmaz, "On the approximate wdisjoint orthogonality of speech," in Acoustics, Speech, and Signal Processing (ICASSP), 2002 IEEE International Conference on, vol. 1, pp. I-529, IEEE, 2002.

[10] A. Liutkus, D. Fitzgerald, Z. Rafii, B. Pardo, and L. Daudet, "Kernel additive models for source separation," IEEE Transactions on Signal Processing, vol. 62, no. 16, pp. 4298-4310, 2014.

[11] D. Fitzgerald, A. Liukus, Z. Rafii, B. Pardo, and L. Daudet, "Harmonic/percussive separation using kernel additive modelling," in Irish Signals \& Systems Conference 2014 and 2014 China-Ireland International Conference on Information and Communications Technologies (ISSC 2014/CIICT 2014). 25th IET, pp. 35-40, IET, 2013.

[12] P. Smaragdis, "Non-negative matrix factor deconvolution; extraction of multiple sound sources from monophonic inputs," in International Conference on Independent Component Analysis and Signal Separation, pp. 494-499, Springer, 2004.
[13] P. Smaragdis, B. Raj, and M. Shashanka, "A probabilistic latent variable model for acoustic modeling," Advances in models for acoustic processing, NIPS, vol. 148, pp. 8-1, 2006.

[14] A. S. Bregman, Auditory scene analysis: The perceptual organization of sound. MIT press, 1994.

[15] F.-R. Stöter, A. Liutkus, R. Badeau, B. Edler, and P. Magron, "Common fate model for unison source separation," in 41st International Conference on Acoustics, Speech and Signal Processing (ICASSP), IEEE, 2016.

[16] T. Chi, P. Ru, and S. A. Shamma, "Multiresolution spectrotemporal analysis of complex sounds," The Journal of the Acoustical Society of America, vol. 118, no. 2, pp. 887-906, 2005.

[17] L. Krishnan, M. Elhilali, and S. Shamma, "Segregating complex sound sources through temporal coherence," PLoS Comput Biol, vol. 10, no. 12, p. e1003985, 2014.

[18] P. Ru, "Multiscale multirate spectro-temporal auditory model," University of Maryland College Park, USA, 2001.

[19] C. Schörkhuber, A. Klapuri, N. Holighaus, and M. Dörfler, "A matlab toolbox for efficient perfect reconstruction time-frequency transforms with logfrequency resolution," in Audio Engineering Society Conference: 53rd International Conference: Semantic Audio, Audio Engineering Society, 2014.

[20] E. Vincent, R. Gribonval, and C. Févotte, "Performance measurement in blind audio source separation," IEEE transactions on audio, speech, and language processing, vol. 14, no. 4, pp. 1462-1469, 2006. 